\title{
DIURNAL VARIATION IN THE OCCURRENCE OF STROKE AND ITS RELATION WITH DIABETES MELLITUS
}

\author{
MIRZA AZIZUL HOQUE ${ }^{1}$, SHAHIDUL ISLAM ${ }^{2}$, MD. SIRAJUL ISLAM ${ }^{3}$, MD. BILLAL ALAM $^{4}$, SM \\ ARIF $^{4}$, ABDUR RAHIM $^{5}$, ASHRAF-UR-RAHMAN ${ }^{5}$
}

\begin{abstract}
:
Objective- To observe diurnal variation in stroke onset and its relation to diabetes mellitus.

Methods - A consecutive series of 139 patients with stroke was admitted at a reference hospitals; the time of onset of symptoms were noted. We compared the diurnal variations between stroke onset both in the diabetic and non-diabetic subjects.

Results - Time of occurrence of stroke was highest in the second quadrant of the 24 hour clock and lowest in the first quadrant. There was no significant association between time of occurrence of stroke and diabetes mellitus

Conclusions - Our study contributes further evidence for a susceptibility stroke event between 6 $A M$ and noon and gives further insights into the possible mechanisms leading to cerebrovascular ischemia. This study also concludes that there is no association between diurnal variation in the occurrence of stroke and diabetes mellitus.
\end{abstract}

Key Words: diurnal variation, stroke onset

\section{Introduction:}

Onset of some diseases including stroke which may cause morbidity and mortality in the individual observed to occur in different part of the day. The diurnal rhythm can be thought of as the mechanism by which the nervous system prepares for different activities throughout the day, metabolism. ${ }^{1}$

Serious adverse cardiovascular events, including myocardial infarction, sudden cardiac death, and stroke, frequently result from rupture of atherosclerotic plaques with superimposed thrombosis and exhibit a pronounced circadian rhythmicity, peaking in the morning hours. Diabetic patients have a very high cardiac event rate. ${ }^{2}$ In this paper we have tried to find out whether there is any relation exists between timing and occurance of stroke along with diabetes mellitus.

\section{Materials and Methods :}

This cross sectional study was carried out between January 2007 and September 2007. 139 consecutive patients (age ranged from 21 to 69 years) who were admitted in the Khulna Medical College Hospital after acute stroke were included in the study. Definition of stroke was based on World Health Organisation criteria.
Patients with transient ischemic attacks, sub-dural haematoma, cortical vein thrombosis or atrial fibrillation were excluded from the study. All patients had a detailed clinical history, physical examination, laboratory studies including blood glucose profile and a CT scan of the brain were done on admission and informed verbal/written consent were taken from the patients/attendants.

At admission, the time of onset of their symptoms was obtained from the patients whenever possible and otherwise were elicited from the attending responsible relatives. We included those patients who/attendant were capable to mention the nearly exact time of occurrence of the stroke with the variation 30 minutes. For patients who had their stroke while asleep, we considered the time of onset to be the hour in which the patient or a relative first became aware of the event. Assuming that the stroke could have occurred at any time during sleep, we also distributed the times of onset between the apparent onset and the hour 11 PM-midnight, when approximately all the patients went to sleep.

Frequency of onset was analyzed for the four 6 -hour intervals in a day. This frequency was compared with the history of diabetes mellitus of the patients. The $x^{2}$ test was done to the number of observed versus

1. Assistant Professor (Endocrinology), Dhaka Medical College, Dhaka.

2. Assistant Registrar, Department of Medicine (unit-1), Khulna Medical College Hospital.

3. Assistant Professor, Department of Medicine, Khulna Medical College

4. Associate Professor, Department of Medicine, Dhaka Medical College, Dhaka

5. Post Graduate Trainee, Department of Medicine, Dhaka Medical College Hospital, Dhaka 
expected strokes during each 6-hour interval. A value of $p<0.05$ was considered statistically significant.

\section{Results :}

We studied 139 patients with stroke (76 men and 63 women) ranging in age from 21 to 69 years (mean age, 63.32 years). Patients had diabetes (29.5\%), hypertension $(65.5 \%)$, family history of stroke in parents $(30.2 \%)$, and history of transient ischaemic attack (30.2\%) (Table I).

Table I

History of diseases in study subjects

\begin{tabular}{lcc}
\hline History of diseases & Frequency & Percent \\
\hline Hypertension & 91 & 65.5 \\
Diabetes Mellitus & 41 & 29.5 \\
Family History of Stroke & 41 & 29.5 \\
in Parents & 42 & 30.2 \\
History of Transient & & \\
Ischemic Attack & & \\
\hline
\end{tabular}

Time of occurrence of CVD was highest in the second quadrant of the 24 hour clock and lowest in the first quadrant (Figure 1).

There was no significant association between times of occurrence of CVD and DM (Table II, Figure 2).

Table- II

Time of Occurrence of CVD and history of Diabetes Mellitus cross tabulation

\begin{tabular}{lccccc}
\hline Time of Occurrence of Stroke & $\begin{array}{c}\text { Diabetes Mellitus } \\
\text { Present }\end{array}$ & $\begin{array}{c}\text { Total } \\
\text { Absent }\end{array}$ & Pearson Chi-Square & P value & \\
\hline 06:01-12:00 & 11 & 37 & 48 & 2.946 & $>0.05$ \\
12:01-18:00 & 10 & 27 & 37 & & \\
18:01-24:00 & 12 & 23 & 35 & \\
24:01-06:00 & 8 & 11 & 19 & \\
\hline Total & 41 & 98 & 139 & \\
\hline
\end{tabular}

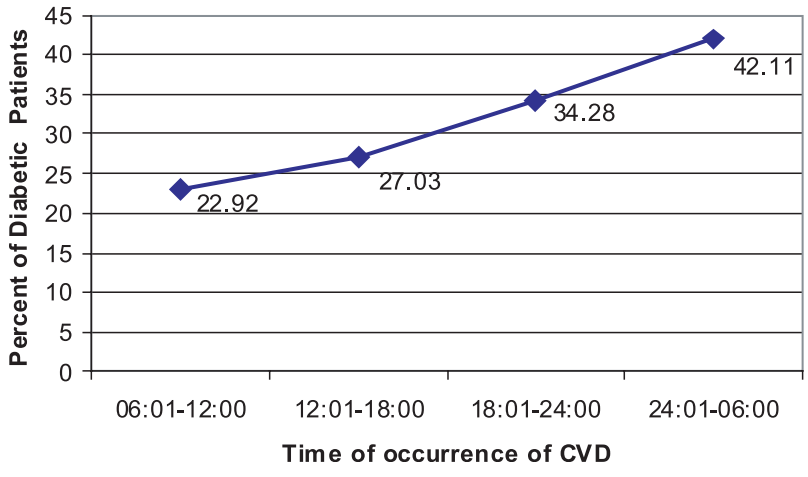

Figure 2: Line graph showing percent of diabetic patients and time of occurrence of CVD

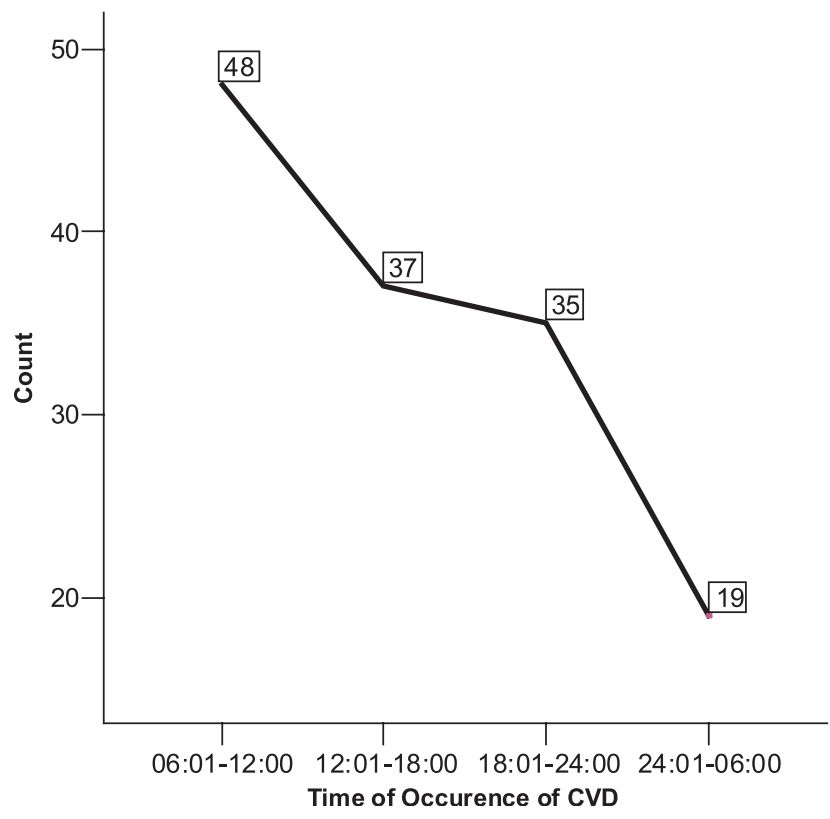

Figure 1: Line graph showing the frequency of occurrence of CVD in different quadrant of the 24 hour clock in a day

\section{Discussion}

Largely controlled by the Suprachiasmatic Nucleus (SCN) of the Hypothalamus, the 24-hour clock is responsible for the release of 'sleepy' hormones around bedtime (i.e. melatonin), morning metabolizing hormones to provide energy after the night fast (i.e. cortisol) and the glucose-metabolizing hormone insulin at appropriate meal times. ${ }^{3}$ Thus, the physiological rhythms of the body are significantly controlled by endogenous factors within the nervous system.

Further neurologically controlled factors that are circadian controlled may disturb and aggravate healthy metabolic functioning and eventually increase 
diabetes mellitus. Cortisol, a hormone involved in the metabolizing of stored energy is released on a 24 -hour rhythm. Like insulin, its secretion correlates with normally anticipated activity levels. Secretion is greatest in the morning while levels drop lowest before sleep. ${ }^{4}$

Insulin sensitivity in patients with Non Insulin Dependents Diabetes Mellitus (NIDDM) changes with circadian (approximately $24 \mathrm{~h}$ ) rhythmicity (decreasing during the night and increasing during the day). These changes are unrelated to blood levels of glucose and insulin, insulin clearance, exercise, food intake, and sleep. They were caused by circadian changes in hepatic glucose production (HGP), which in turn were closely correlated with circadian changes in blood Free fathy acid and cortisol levels. ${ }^{5}$ Our data show that stroke onset is not random during 24 hours but that it has a characteristic distribution. During the interval from 06:01 to 12:00 hour, 34.5\% $(n=48)$, from $12: 01$ to $18: 00$ hour $26.6 \%(n=37)$, from $18: 01$ to 24:00 hour 25.2\% ( $\mathrm{n}=35)$ and from 24:01 to 06:00 hour $13.7 \%(\mathrm{n}=19)$ patients were attacked by CVD. This finding is similar to the finding of Lago et al. ${ }^{6}$ Marshall ${ }^{7}$ in his study excluded embolic strokes, resulting in a selection bias. In that series, cerebral ischemic events showed a higher incidence of onset between midnight and $6 \mathrm{AM}$. Hossman ${ }^{8}$ assumed that in the presence of stenosis of the extracranial and intracranial vessels, similar hemodynamic mechanisms were the likely cause of the event in the majority (30\%) of his patients who had their strokes between 1 and $5 \mathrm{AM}$.

Our study findings showed that the circadian variation of occurrence of stroke is not associated with the history of diabetes mellitus in the patients. This finding is consistent with the findings of Lago et al. ${ }^{6}$ and Argentine et al. ${ }^{9}$ Alterations in the circadian variation autonomic tone, blood pressure, and the thrombotic-thrombolytic equilibrium have been documented in diabetic patients. These include reduced or absent 24 -h periodicity in autonomic tone, fibrinolytic activity, and thrombotic tendency, and a blunted decline in nocturnal blood pressure. Disruption of these circadian rhythms explains the lack of significant circadian distribution of cardiac events in diabetic patients. ${ }^{5}$

\section{Conclusion:}

Our study contributes further evidence for a susceptibility stroke event between $6 \mathrm{AM}$ and noon and gives further insights into the possible mechanisms leading to cerebrovascular ischemia. This study also concludes that there is no association between circadian pattern of occurrence of stroke and diabetes mellitus.

\section{References:}

1. Kate Matney. Circadian Rhythms in Shift Workers and Diabetes. In: Biology 202, [Online]. Available at: http://serendip.brynmawr. edu/bb/neuro/neuro05/ web3/kmatney.html. Accessed spring 2005.

2. Scott Alene. Shift Work Hazards. In: Jacksonville Medicine. Available at: www.dcmsonline.org/jaxmedicine/2001journals/April2001/shiftwork.htm. Assessed April, 2007.

3. Nissl J, Houten SV, Landauer T, Rhoads, Caroline S, Dalkin AC. In: Cortisol in Blood. Available at: my.webmd.com/hw/health_guide_atoz/hw6227.asp. Accessed August 2006.

4. Boden G, Chen X, Urbain JL. Evidence for a circadian rhythm of insulin sensitivity in patients with NIDDM caused by cyclic changes in hepatic glucose production. Diabetes 1996; 45:1044-1050.

5. Aronson Doron. Impaired modulation of circadian rhythms in patients with diabetes mellitus: A risk factor for cardiac thrombotic events? Chronobiology international 2001; 8(1): 109-121.

6. Lago A, Geffner D, Tembl J, Landete L, Valero C, Baquero M. Circadian Variation in Acute Ischemic Stroke: A Hospital-Based Study Stroke 1998; 29: 1873-1875.

7. Marshall J. Diurnal variation in occurrence of strokes. Stroke1977; 8: 230-231.

8. Hossman V. Circadian changes of blood pressure and stroke. In: Zulch KJ (ed). Cerebral Circulation and Stroke. Berlin/Heidelberg/New York: Springer, 1971: 204-208.

9. Argentine C, Toni D, Rasura M, Violi F, Sacchetti ML, Allegretta A et al. Circadian Variation in the Frequency of Ischemic Stroke. Stroke. 1990; 21: 387389 . 